\title{
The Development of a Multi-frequency Electromagnetic Instrument for Monitoring the Phase Transformation of Hot Strip Steel
}

\author{
S.J. Dickinson ${ }^{1}$, R. Binns ${ }^{1}$, W. Yin ${ }^{1}$, C. Davis ${ }^{2}$, A.J. Peyton ${ }^{1}$ \\ ${ }^{1}$ Enginerring Department, Lancaster University, Lancaster LA1 4YR, UK \\ ${ }^{2}$ Dept Met \& Mat, Univ. Birmingham, Birmingham B15 2TT, UK \\ Email: wuliang.yin@manchester.ac.uk
}

\begin{abstract}
This paper describes the development of an instrument to analyze the phase transformations of hot strip steel using an electromagnetic sensor. The sensor exploits variations in the electrical conductivity and magnetic permeability of the steel to monitor microstructure evolution during processing. The sensor is an inductive device based on a H-shaped ferrite core, which is interrogated with a multi-frequency impedance analyzer (MFIA) containing a digital signal processor (DSP). An overview of the instrument and measurements from a range of carbon steels sample are presented. The results verify the ability of the instrument both to monitor the micro-structural changes and to reject variations in liftoff distance between the sensor and the hot strip.
\end{abstract}

\section{INTRODUCTION}

In the production of steel strip, the temperature distribution and cooling rates have a significant effect on the steel microstructure and hence on final material properties, such as yield strength, tensile strength and ductility. Increasingly, steel customers require better materials in order to improve their own products and processing lines that use automatic feeding or similar techniques. Consequently, demands for higher product consistency and a greater diversity of sophisticated steel grades have increased the requirements for tighter control of process conditions and hence improved instrumentation. The aim of this paper is to describe a new instrument to monitor the microstructure of steel during controlled cooling on a hot strip mill.

\section{BACKGROUND}

An overview of the application is shown in Figure 1. When hot steel strip leaves the last rolling stand, the structure is usually the high temperature, face-centered cubic austenite phase. As it cools, it transforms to a structure consisting of the body centered cubic ferrite phase and carbide, usually cementite $\left(\mathrm{Fe}_{3} \mathrm{C}\right)$, the morphology of the latter depending on cooling rate and composition. Increasing the cooling rate or alloy content causes transformation to occur at lower temperatures giving a finer carbide dispersion and hence a stronger product. By altering the final microstructures a wide range of strengths can be produced in the strip product from very low carbon essentially ferritic structures with tensile strengths of about $200 \mathrm{~N} / \mathrm{mm}^{2}$ to high strength steels with tensile strengths in excess of $1000 \mathrm{~N} / \mathrm{mm}^{2}$. These have higher carbon contents with microstructures consisting of mixtures of ferrite, pearlite, bainite, martensite and, in some cases, known as TRIP steels, austenite which by suitable alloying has been stabilized at temperatures down to ambient.

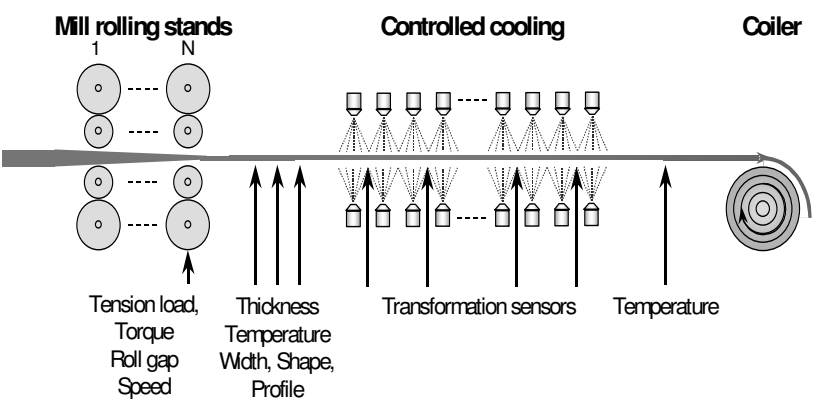

Figure 1. The schematic diagram of a hot strip mill

Non-contacting optical temperature sensors are typically used to implement feedback control of cooling. Unfortunately, water spray variations and surface emissivity irregularities adversely affect these sensors. The optical pyrometers are therefore positioned outside the quenching zone and mathematical models are used to interpolate the condition of the steel strip during cooling [1-3]. In addition, temperature is only used as an assumed indicator of microstructure and only the surface of the steel is measured. Ideally, the control of cooling path should take account of the progress of dynamic transformation at a particular point rather than the strip temperature alone. A transformation monitoring system used in conjunction with optical pyrometers would therefore represent a significant step forward.

A number of approaches have been proposed and studied for monitoring transformation on-line, including X-ray diffraction [4], X-ray attenuation to monitor density change [5], ultra-sonic [6], thermal and magnetic. It is well known that the electromagnetic (EM) properties of steel change significantly as the material progresses through phase transformation [7]. The austenitic phase is paramagnetic and the ferritic phase is ferromagnetic below the Curie point, which is around $770{ }^{\circ} \mathrm{C}$ but depends on the chemical composition of the steel. The iron-carbon phase diagram, see Figure 2(a), shows the temperatures of the main transformations versus the carbon content, when cooled very slowly. Any austenite to ferrite transformation occurring below the Curie temperature may be detected by the ferromagnetic transformation. However, for $\mathrm{Fe}-\mathrm{C}$ alloys with 
a carbon content less than $0.6 \mathrm{wt}$. $\%$, the austenite-to-ferrite transformation starts above the Curie temperature. The ferrite thus formed remains paramagnetic and above the Curie temperature this phase change would be difficult to detect magnetically, unless very sensitive devices were used. However, in practice, common alloying additions and accelerated cooling of the steel on the run-out tables of strip mills reduces the austenite-to-ferrite transformation temperature to below the Curie temperature, in most cases, except at very low carbon contents. For example, Figure 2(b) shows a continuous cooling transformation diagram for a $0.74 \% \mathrm{C}$ steel where even at very slow cooling rates (e.g. 0.4 ${ }^{\circ} \mathrm{C} / \mathrm{s}$ ) transformation from austenite does not take place above $700{ }^{\circ} \mathrm{C}$. The phase change is indicated by the bump in the temperature plots caused by the release of the latent heat of transformation. Thus, micro-structural changes can be detected and monitored on-line using magnetic sensors. Phase transformations have been previously studied using thermal analysis techniques [8] and more widely by dilatometry but these are essentially laboratory techniques and are not suitable for on-line use.

\section{Temperature ${ }^{\circ} \mathrm{C}$}

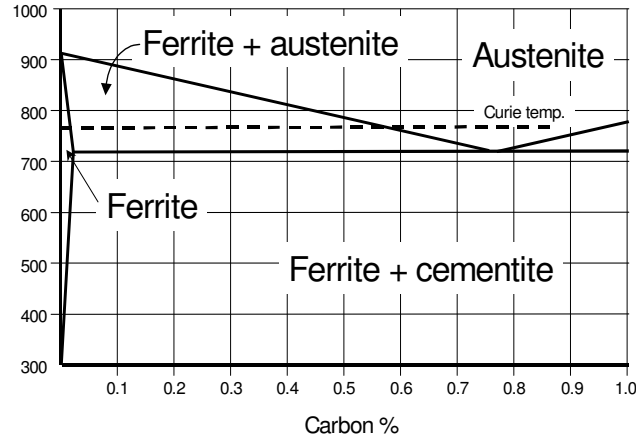

(a)

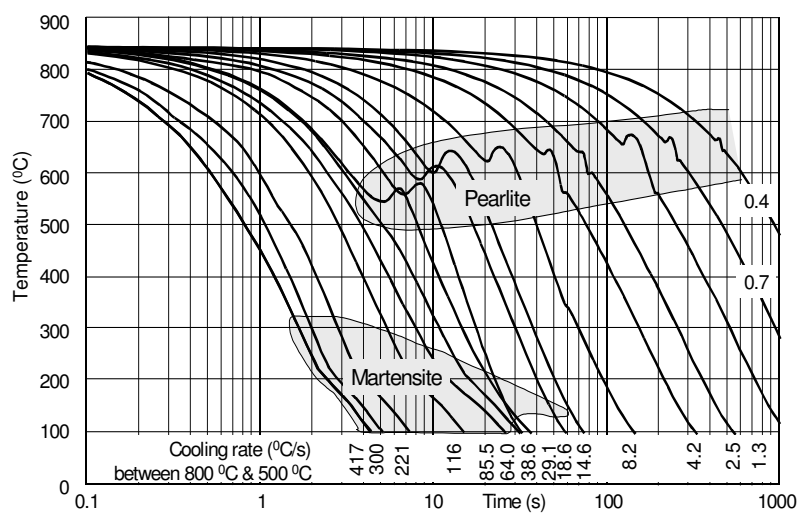

(b)

Figure 2. Transformation data. a) Fe-C phase diagram (b) Continuous cooling transformation diagram for a $0.74 \% \mathrm{C}$ steel

The electromagnetic approach has several potential advantages. The magnetic field is unaffected by water and any non-magnetic, non-conductive material used to protect the sensor. In addition, the field acts at a distance and requires no contact with the hot strip. There are several reports of the successful use of electromagnetic sensors to monitor transformation both in the laboratory and on the runout table [9-17]. These systems used either DC or a single excitation frequency. Consequently, separate measurements are required to compensate for variations in the distance (or lift-off) between the sensor and strip steel. The multifrequency approach in the paper allow the phase signature of the signal spectrum to be used directly, which has been shown to be approximately lift-off independent. The analytical treatment of the topic is dealt with in a separate paper by Yin et al [18].

Recently, research has also been focused the underlying relationship between the microstructure of the steel and the electromagnetic response. In particular, the sensor output has been shown to have a monotonic, non-linear response to the ratio of transformed ferrite, which is dependant on the morphology of the ferromagnetic phase [19]. Finite element simulation at the microstructure level has also been used to model the experimental results [20]. Surface conditions of the steel such as the presence of decarburization can significantly affect the response of the sensor [21] although in practice on a full scale mill this is not considered to present a difficultly as the surface will not have time to react with the atmosphere before being cooled.

\section{SYSTEM DESCRIPTION}

An overview of the system is shown in Figure 3. The acquisition system contains a digital signal processor (DSP), impedance measuring circuitry and a sensing head. The DSP has dedicated on-board firmware, which operates in response to commands received via a serial link from the host computer. The DSP controls the measurement process and can generate a composite excitation waveform containing up to 8 frequencies in a binary sequence. The excitation waveform is fed to the sensing head via a digital-to-analogue converter (DAC) and buffer amplifier. The impedance measurements are achieved using two analogue-to-digital converters (ADC's), one for measuring the excitation current and one measuring the detected voltage. An important design issue is the ability to measure a small impedance change superimposed on a large background, which is often the case in electrical tomography. Consequently, a second ADC is used to null the background signal. 


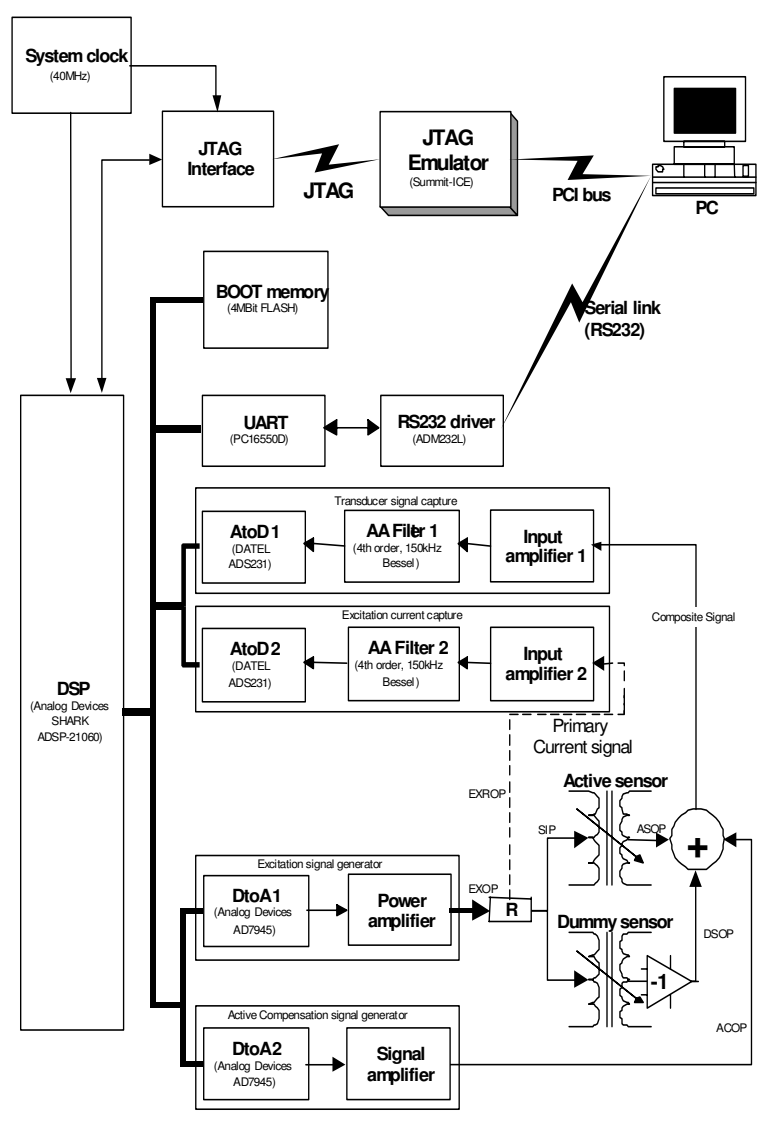

Figure 3. Block diagram of the system hardware

The magnetic sensing head consists of a $\mathrm{H}$-shaped ferrite core, $115 \mathrm{~mm}$ high, $100 \mathrm{~mm}$ wide with a $25 \mathrm{~mm}$ square cross section, shown in Figure 4(a) before encapsulation. The sensor geometry was designed to enable a lift-off of between $10 \mathrm{~mm}$ and $100 \mathrm{~mm}$. The sensing head has five coils; a primary excitation coil and four secondary flux sensing coils as shown in Figure 4(b). Essentially the arrangement may be thought of as two $U$ cores joined back to back with a common excitation coil, one of which being used as the active sensing element and the other as a dummy reference element. Adjacent pairs of secondary pickup coils are wired in series so maximizing the signal of interest while helping reduce common mode interference caused by ambient magnetic fields.

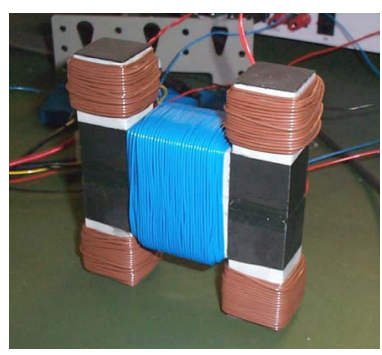

(a)

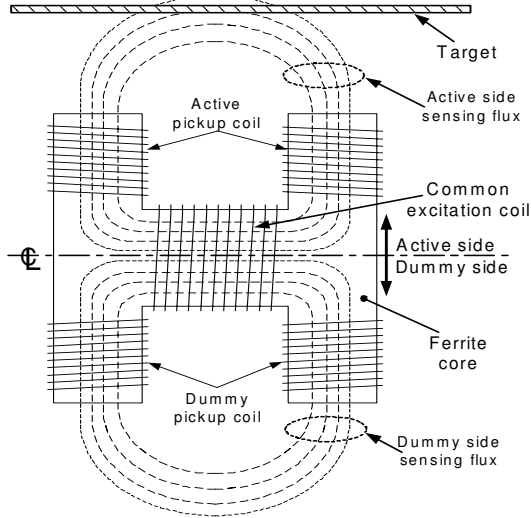

(b)

Figure 4. The magnetic head before encapsulation (a) and the main elements of the magnetic sensing head (b)

Multi-frequency impedance analysis is performed by exciting the sensing head with a composite waveform containing the required harmonics, each at a nominal phase and magnitude. The actual excitation current and the flux sensing coils' voltages are sampled and analyzed by the DSP using a fast Fourier transform (FFT) to extract the harmonic phase and magnitude information, from which associated impedances are calculated. To maximize the dynamic range of the system, it is necessary to null the background voltage from active sensing element's output voltage. The background voltage can be several orders of magnitude greater than the desired signal from the steel and must be carefully removed. A three-tier approach is used. First, most of the unwanted voltage is removed by subtracting the output voltage from head's dummy sensing element from that of the active head using a magnetic bridge configuration as described above. Second, the residual voltage, which is caused by differences between the active and dummy sides of the sensor, is subtracted using a compensation waveform generated from a second ADC. A multi-frequency compensation waveform is required as the residual has frequency dependent magnitude and phase errors. Finally, any further residual of the background signal is subtracted in software.

The compensation waveform was determined in the following three stages.

1. The magnitude and phase response of the sensor was measured,

2. The response of the compensation channel was determined,

3. The required compensation wave was calculated.

The purpose of the first stage is to characterize the frequency magnitude and phase response of the entire activecompensation loop. This loop starts at the active compensation DAC's output and passes through the inputsumming amplifier, gain amplification stages and filtering and ends at the head voltage sensing ADC (analogue to digital converter) as shown in Figure 3. The test is performed 
using small, harmonic rich, test wave as described by equation 1, to drive the loop via the active compensation DAC. The head excitation wave is also set to zero.

$$
x_{t}=\sum_{n=1}^{8}\left|\mathbf{X}_{n}\right| \sin \left(\arg \left(\mathbf{X}_{n}\right)+2^{(n-1)} \omega t\right)
$$

where $\mathbf{X}$ is a vector containing eight complex harmonic components and $x$ is the active compensation DAC's output voltage (the loop input voltage). The resulting voltage at the end of the loop is then sampled by the voltage sensing ADC and analyzed using an FFT to obtain the complex frequency components $\mathbf{Y}$. The vector $\mathbf{A}$ describing the loop gain and phase response at each discrete frequency is then calculated as shown by equation 2 , which characterizes the active compensation loop.

$$
\mathbf{A}=\frac{\mathbf{Y}}{\mathbf{X}}
$$

The second step is to measure the residual voltage from the sensing head. This is done by setting the active compensation wave to zero, applying the normal excitation wave and then sampling the voltage sensing ADC. The residual voltage is then analyzed by means of an FFT in order to obtain the complex frequency components $\mathbf{W}$. The third and final stage involves calculating the voltage wave required to null the unwanted residual voltage wave. The final compensation wave harmonic vector $\mathbf{C}$ is as described by equation 3.

$$
\mathbf{C}=\frac{-\mathbf{W}}{\mathbf{X}}
$$

Further details on the MFIA are presented elsewhere [22].

\section{RESULTS}

Three sets of steel samples of compositions given in Table 1 and dimension $137 \mathrm{~mm}$ x $87 \mathrm{~mm}$ x $2 \mathrm{~mm}$ were investigated in order to verify the effects of phase transformations and changes in lift-off. Each sample was heated to $1000{ }^{\circ} \mathrm{C}$ and then placed over the sensor and allowed to cool in free air.

Table 1: Compositions of steels under investigation

\begin{tabular}{|c|c|r|r|r|r|}
\hline & \multicolumn{5}{|c|}{ COMPOSITION (\% wt) } \\
\hline Sample & C & \multicolumn{1}{|c|}{ Si } & Mn & P & S \\
\hline Low Carbon (A) & 0.09 & 0.02 & 0.94 & 0.017 & 0.003 \\
\hline $\begin{array}{c}\text { Medium carbon } \\
\text { (B) }\end{array}$ & 0.44 & 0.25 & 0.81 & 0.017 & 0.005 \\
\hline \begin{tabular}{c} 
High Carbon (C) \\
\hline
\end{tabular} & 0.68 & 0.22 & 0.74 & 0.009 & 0.001 \\
\hline
\end{tabular}

The samples were thermally insulated within a $1 \mathrm{~cm}$ thick Duratec enclosure, which provided a slow controlled cooling rate $\left(<1{ }^{\circ} \mathrm{C} / \mathrm{s}\right)$, hence near equilibrium transformation was obtained. The measured temperature uniformity across a sample was less than $10^{\circ} \mathrm{C}$.
Two plots of the changes in the measured impedance versus temperature for the low carbon (A) and high carbon (C) samples are shown in Figure 5 for a lift-off of $51 \mathrm{~mm}$. Each graph contains 8 curves for excitation frequencies ranging from $781 \mathrm{~Hz}$ to $100 \mathrm{kHz}$ in a binary sequence of values, as indicated in the legend.

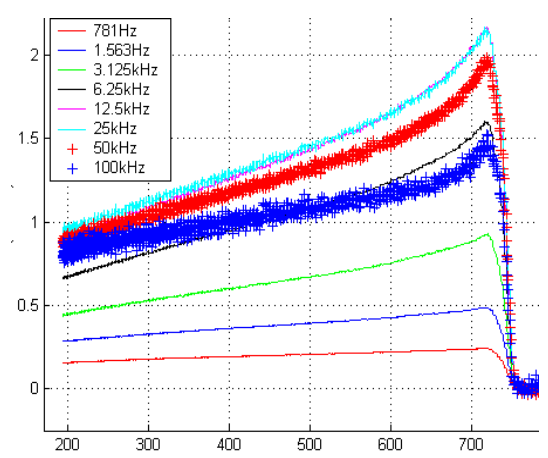

(a)

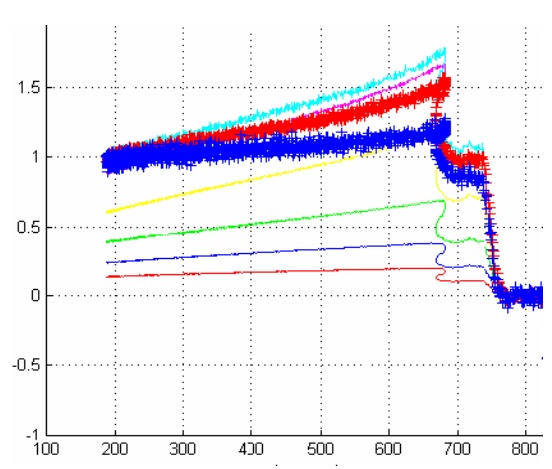

(b)

Figure 5. Changes in the measured trans-impedance (ohms) versus temperature $\left({ }^{\circ} \mathrm{C}\right)$ for the low carbon (a) and high carbon (b) samples

For the low carbon sample (A), the curves all display a steep rise at the Curie temperature, $T_{C}$, which is the point where the material becomes ferromagnetic. After this transformation, (i.e. below $700{ }^{\circ} \mathrm{C}$ ) the low frequency curves (e.g. $781 \mathrm{~Hz}$ ) are relatively flat. As the frequency increases and samples cool, the curves reduce in value because the interrogating EM field cannot diffuse as deeply into the strip. The depth of penetration, $\delta$ to approximately $e^{-1}$ of the surface value, is governed by the skin depth equation,

$$
\delta=\sqrt{\frac{2}{\mu \cdot \omega \cdot \sigma}}
$$

where $\mu$ is the magnetic permeability and $\sigma$ is the electrical conductivity of the sample and $\omega=2 \pi f$ is the angular frequency. As the sample cools, both conductivity and permeability increase and consequently the interrogating field can only penetrate less of the thickness of the sample. This effect is more pronounced for higher frequencies. 
For the high carbon sample (C), the curves show the same trends, but with the addition of a second transformation at the eutectoid temperature $\left(680{ }^{\circ} \mathrm{C}\right)$, caused by the presence of the carbon. This effect is shown in more detail in Figure 6, with a lift-off of $51 \mathrm{~mm}$ and an excitation frequency of $21.5 \mathrm{kHz}$.

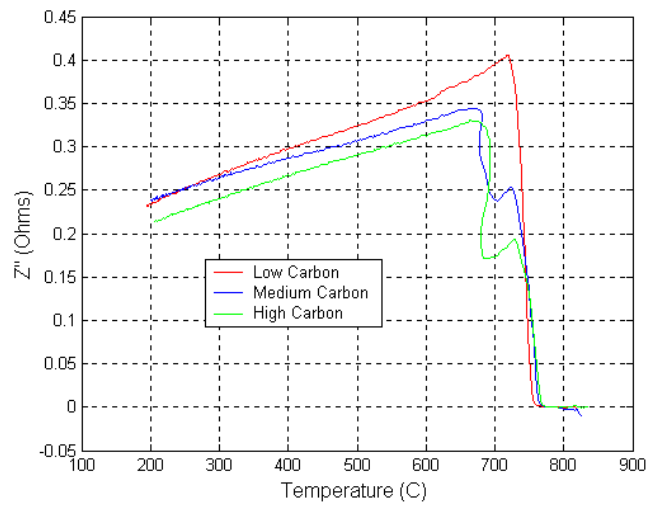

Figure 6.Influence of carbon composition upon imaginary impedance Z" at $12.5 \mathrm{KHz}$

Sample A shows an increase in the impedance at $\mathrm{T}_{\mathrm{C}}(\sim$ $\left.770{ }^{\circ} \mathrm{C}\right)$. Due to its low carbon content, this sample is expected to transform fully to ferrite above $T_{C}$, so here the sensor is detecting the paramagnetic to ferromagnetic transformation. Samples B and C contain more carbon $(0.44$ and $0.68 \%$ wt respectively) and are expected to transform to a mixed ferrite and pearlite microstructure, with the majority of the ferrite forming above $\mathrm{T}_{\mathrm{C}}$ and the pearlite forming at the eutectoid temperature, $\mathrm{T}_{\mathrm{E}},\left(\sim 723{ }^{\circ} \mathrm{C}\right.$ at equilibrium, but suppressed to $\sim 680{ }^{\circ} \mathrm{C}$ by the cooling rate used). The higher carbon content of the sample $\mathrm{C}$ results in a higher pearlite and by implication lower ferrite content. Hence a larger expected $Z$ " response at $T_{E}$ and a lower $Z$ " change at $T_{C}$ as shown in Figure 6. Differences in measured impedance therefore imply discrimination between steels of varying carbon composition, as seen in previous work [19].

The effect of lift-off is shown in Figure 7, which plots the magnitude of the trans-impedance change (in $\Omega$ ) and change in phase (deg.) versus both temperature $\left({ }^{\circ} \mathrm{C}\right)$ and lift-off $(\mathrm{mm})$. Changes in magnitude are shown on the left hand side and phase changes are shown on the right, with an excitation frequency of $32 \mathrm{kHz}$. These curves were obtained by testing a series of samples cut from adjacent positions on the sample and therefore with virtually identical material properties. Lift-off distances of between 42 and $70 \mathrm{~mm}$ were considered in $3 \mathrm{~mm}$ increments. Each sample was only used for one hot test. The effect of lift-off is generally to simply scale the size of the magnitude, the larger the lift-off the smaller the signal. However, lift-off has little effect on the phase response as these curves are virtually constant in this direction. This was found to be the case for all the measured frequencies, which suggests that the phase signature of the trans-impedance is approximately lift-off independent. The theoretical basis of this result is treated in a complementary paper [18].

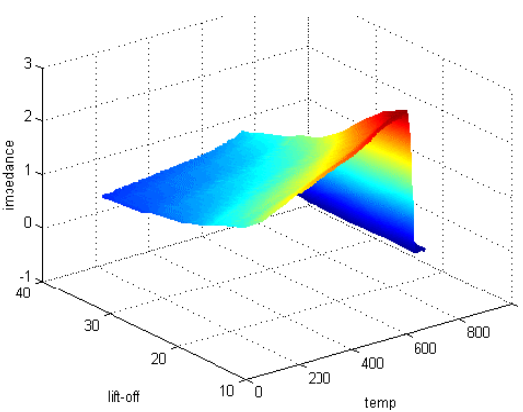

(a)

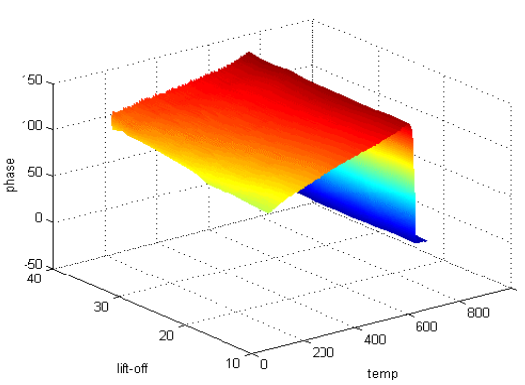

(b)

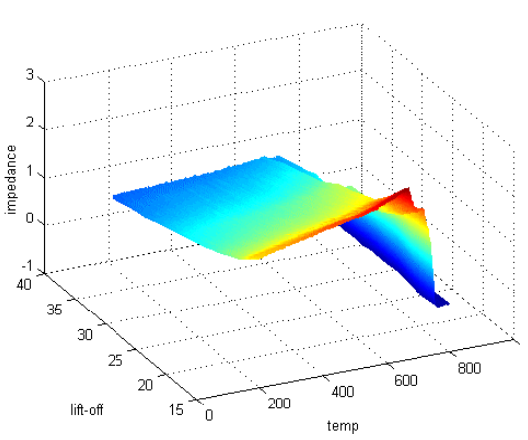

(c)

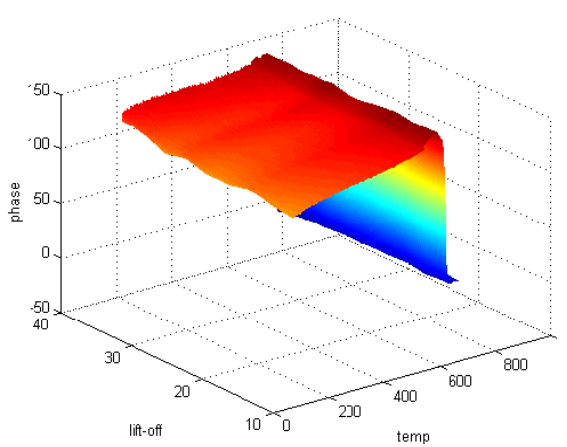

(d) 


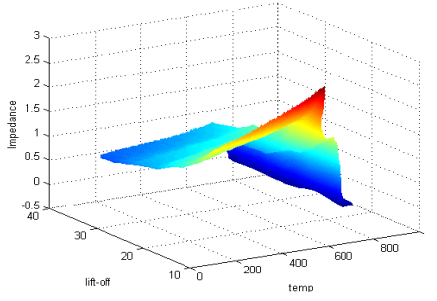

(e)

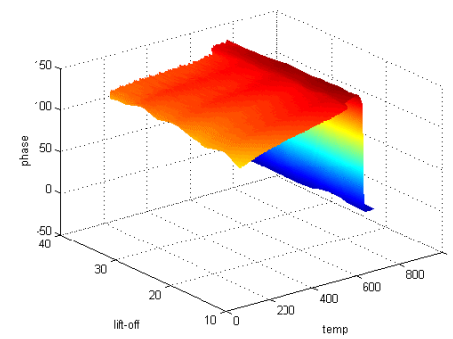

(f)

Figure 7. Effect of lift-off changes on the sensor response at $32 \mathrm{kHz}$. Figures (a) and (b) are the magnitude $(\Omega)$ and phase (deg) for sample A respectively. Figures (c) and (d) are the magnitude $(\Omega)$ and phase (deg) for sample B respectively. Figures (e) and (f) are the magnitude $(\Omega)$ and phase (deg) for sample $\mathrm{C}$ respectively.

\section{CONCLUSIONS}

This paper has described the design and operation of an instrument to analyze the phase transformations of hot strip steel using electromagnetic interrogation. The instrument uses a novel multi-frequency excitation method to monitor the micro-structural changes. The paper has shown that the instrument can successfully detect transformation at both the Curie and Eutectoid temperatures. Furthermore, the sensor is able to discriminate the response between steels of varying carbon composition. The measurements presented in the paper confirm the phase response of the sensor is virtually lift-off independent. This is the first time this result has been reported with tests on hot steel samples.

\section{ACKNOWLEDGMENT}

The authors wish to express their gratitude to EPSRC and for its financial support of this project.

\section{REFERENCES}

[1] (NISI ) Nissan Steel KK, "Controlling Phase Transformation in Coiled Hot Rolled Steel Strip by Monitoring Temp. on Leaving Hot Rolling Mill and Applying Cooling Water in Computer Calculated Amount", WPI Acc. No. 77-65980Y/37, 1977.
[2] Masayoshi Suehiro, Takehide Senuma, Takashi Oda, Seiji Konishi., "Development Mathematical Models for Predicting Transformation of High-Carbon Steel During Cooling on Run-out Table and its Application to On-line Temperature Control of Hot Strip Mill”, Nippon Steel Technical Report, No. 67, October 1995.

[3] A.A. Tseng, J.G. Chang, M. Raudensky, J. Horsky, "An Inverse Finite Element Evaluation of Roll Cooling in Hot Rolling of Steels", J. Mat. Proc. And Man. Sci., Vol. 3(4), pp. 387-408, 1995.

[4] H. Kitagawa and T. Sohmura, "An X-ray diffraction method for quantitative determination of retained austenite in the production line of metastable austenitic stainless steel". Trans. ISIJ, Vol. 23, pp. 543-9, 1983.

[5] P. Gaujé, "Measure de densité par absorption de rayonnements ionisants. Application à la caractérisation des aciers". La Revue de Métallurgie CIT, pp. $1145-50,1990$.

[6] M. Dubois J. F. Bussiere, "Rapid microstructure assessment in rolled steel products using laser ultrasonics", Non Destr. Charact. of Materials, pp. 329-333, 1998.

[7] R. Bozorth, "Ferromagnetism", Van Nostrand, New York, 1951.

[8] A. Bodin and P.D. Marchal, "The use of thermal analysis techniques for the validation of phase transformation models", Steel Res. Vol. 65(3), pp. 103-9, 1994.

[9] Inst. Rech Siderurgie, "Hot-Rolling Thick Steel Plate on a Four-High Mill using Detectors to Indicate Start of Gamma-Alpha Transformation”, WPI Acc. No. 75-79163W/48, 1975.

[10] Inst. Rech Siderurgie, "Cooling of Steel Wire - Includes Detection of the Gamma-Alpha Transformation", WPI Acc. No. 78-62228A/35, 1978.

[11] N. Ishibashi, M. Morita, K. Sakata, K. Hashiguchi, and S. Okano, "Method for measuring strip temperature with magnetic sensor", Trans. ISIJ, 27, p B-54, 1987.

[12] M. Morita, K. Hashiguchi, O. Hashimoto, H. Nishida, and S. Okana, "Development of on-line transformation detector for property control of hot rolled steel", Proc. Mat. Soc. AIME Symposium on Accelerated Cooling of Steel, Pittsburgh, Aug. 1987.

[13] K. Yahiro, J. Yamasaki, M. Furukawa, K. Arai, M. Morita, and M. Obashi, "Development of coiling temperature control system on hot strip mill", Kawasaki Steel Giho, Vol. 22(1), pp. 12-18, 1990.

[14] Han-Soo Kim, Il-han Park, Sang-Bong Wee, Song-Yop Hahn, and Min-Koo Han, "Eddy current methods for evaluating the transformed fraction of metals by voltage source", IEEE Trans. Mag., Vol. 26(2), pp. 505-8, 1990 .

[15] P. Gauje and S. Segalini, "Detection on-line du point de transformation Alpha-Gamma", Commission des Communautes Europeenes, Convention no. 7210 GB/307, MCA.RCA 91318, September 1991.

[16] R. Binns, S. Johnstone, S. Mallinson, T. Meydan, A.J. Peyton, W.D.N. Pritchard, and T. Walker. "Using Electromagnetic Methods to Monitor the Transformation of Steel Samples", Institute of Materials Conf., 27 28 April 1999, London.

[17] S. Johnstone, R. Binns, A.J. Peyton, and W.D.N. Pritchard, "Using electromagnetic methods to monitor the transformation of steel samples", Trans. Inst. Measurement \& Control, Vol. 22, 2000.

[18] W. Yin, R. Binns, C. Davis, and A.J. Peyton, "Analysis of the lift-off effect of phase spectra for eddy current sensors" Submitted to IEEE IMTC2005

[19] C.L. Davis, M.P. Papaelias, M. Strangwood, and A.J. Peyton, "Measurement of phase transformations in steel using electromagnetic sensors". J.Iron. Steel. Vol 29(6), pp.469-76, 2002.

[20] M.P. Papaelias, M. Strangwood, A. J. Peyton, and C.L. Davis, "Detection and measurement of phase transformation in steels using electromagnetic sensors - Experimental results and modelling simulations" Met. Trans. A. In press ??.

[21] M. Ph. Papaelias, M. Strangwood, A. J. Peyton, C. L. Davis, "Effect of microstructural variations on smart inductive sensor measurements of phase transformation in steel" Pending publication ?? Scripta Metallica

[22] S. Dickinson and A. J. Peyton, "A high speed, multi-frequency impedance analysing node." 2nd World Congress on Industrial Process Tomography, Hanover, ISBN 0-85316-224-7, pp. 502-510, 29 Aug. to 1 Sept. 2001. 